\title{
Solution-Liquid-Solid Approach to Colloidal Indium Nitride Nanoparticles from Simple Alkylamide Precursors
}

\author{
Niladri S. Karan, Yang Chen, Zhihui Liu, Rémi Beaulac* \\ Department of Chemistry, Michigan State University, East Lansing, Michigan 48824-1322, United States
}

\section{Table of Contents}

1. InN Nanocrystal Synthesis p. 1

2. Characterization p. 2

3. TEM images and size distribution analyses

$\begin{array}{ll}\text { a. As-prepared sample p. } 3 & \text { p. } 12\end{array}$

$\begin{array}{ll}\text { b. Acid-treated sample } & \text { p. } 12\end{array}$

c. InN NRs synthesized from lithium bis(trimethylsilyl)amide (LiHMDS) p. 22

$\begin{array}{ll}\text { 4. XPS data } & \text { p. } 24\end{array}$

5. Raman data $\quad$ p. 24

6. Absorption data, comparison with the $\mathrm{NaNH}_{2}$ synthesis p. 25

7. EDS data of the InN/In(0) sample p. 25

$\begin{array}{ll}\text { 8. References } & \text { p. } 26\end{array}$

\section{InN Nanocrystal Synthesis}

All solvents were purchased from Aldrich or Fischer, ACS grade or better unless otherwise noted. Reactions were performed in a nitrogen-filled glovebox or on a Schlenk line under positive nitrogen pressure. $\mathrm{InBr}_{3}$ (Alfa Aesar, 99.99\%), hexadecane (Sigma Aldrich, 99\%), 1-octadecene (ODE, Acros, $90 \%$ ), oleylamine (OLA, Sigma-Aldrich, $\geq 98 \%$ ), $n$-butyllithium solution, $2.5 \mathrm{M}$ in hexane ( $n$-BuLi, Sigma Aldrich), $N, N, N^{\prime}, N^{\prime}$-tetramethylethylenediamine (TMEDA, Sigma Aldrich, 99\%), hexamethyldisilazane (HMDS, referred to as "bis(trimethylsilyl)amine" in the text, Alfa Aesar, 99+\%), trichloroethylene (TCE, Sigma-Aldrich, $>99.5 \%$ ), ethanol (EtOH, Macron), nitric acid $\left(\mathrm{HNO}_{3}, \mathrm{EMD}\right)$, chloroform $\left(\mathrm{CHCl}_{3}\right.$, Macron), and toluene (Macron). All compounds were used as received unless otherwise noted. The solvents (1-octadecene and hexadecane) were degassed and dehydrated overnight at $80{ }^{\circ} \mathrm{C}$ under dynamic vacuum $(<10 \mathrm{mT}$ Torr) before being transferred to and stored in the glovebox.

In a three-neck round-bottom flask equipped with a water-cooled-condenser and kept under air-free conditions $\left(\mathrm{N}_{2}\right.$ Schlenk line $), 180 \mathrm{mg} \mathrm{InBr} 3(0.5 \mathrm{mmol})$ are solubilized in $2 \mathrm{ml}$ of oleylamine $(6.39 \mathrm{mmol})$ and $5 \mathrm{ml}$ of a high-boiling point aliphatic solvent (octadecene or hexadecane: we did not observe any difference whatsoever in using either solvents). A complete dissolution of the salt is achieved by heating the solution to $220^{\circ} \mathrm{C}$ under constant vigorous stirring. An alkylamide precursor solution is separately prepared under air-free conditions $\left(\mathrm{N}_{2}\right.$ glove box $)$ by reacting $0.50 \mathrm{ml}$ of OLA $(1.5 \mathrm{mmol})$ with a mixture of $0.60 \mathrm{ml}$ of a $n$-BuLi solution ( $2.5 \mathrm{M}$ hexane solution, $1.5 \mathrm{mmol} n$-BuLi) and $0.45 \mathrm{ml}$ TMEDA ( $3 \mathrm{mmol})$. This alkylamide solution is then injected promptly with a $6 \mathrm{ml}$ syringe into the indium-OLA mixture to initiate the reaction. After injection, the temperature of the indium solution drops to $175 \pm 2{ }^{\circ} \mathrm{C}$ and quickly (less than a minute) rises back to $210^{\circ} \mathrm{C}$, where it is maintained until the reaction is stopped. The solution changes from colorless to brownish-black over the course of the first few minutes, indicative of the formation of $\mathrm{InN}$ nanocrystals. The reaction is stopped after 10 minutes by removing the heat source and letting the solution cool down to room temperature. The resulting solution is then sonicated in ethanol $(15 \mathrm{ml})$ for two minutes, followed by a five minutes centrifugation at $4,000 \mathrm{rpm}$ to crash out the solids; this sonication/centrifugation cycle is then repeated a second time, after which the sample is dissolved in a nonpolar solvent, toluene or chloroform for routine storage or measurements, or tetrachloroethylene for NIR spectroscopy. The sample is either analyzed directly ("as-prepared"), further functionalized with 
oleylamine as described below ("as-prepared, functionalized"), or further purified to eliminate $\operatorname{In}(0)$ from the sample. For the latter, the as-prepared sample is sonicated in nitric acid $(15 \mathrm{ml}, 3 \% \mathrm{vol})$ for two minutes then centrifuged at 4,000 rpm for 5 minutes. The resulting sample ("acid-treated") is then washed following the same protocol described above. The sample can be directly converted into a stable colloidal suspension by heating the solid in $3 \mathrm{ml}$ OLA at $80^{\circ} \mathrm{C}$ for 3 hours. The resulting mixture is then washed by sequential ethanol/toluene treatments to yield a toluene (or another non-polar solvent) suspension of InN nanoparticles that remains stable for days without signs of solids crashing out of the solvent. Samples synthesized using other amide precursors were made following the same approach, but substituting a different amine in the deprotonation step above, such as HMDS. For measurements of the evolution of the reaction, small aliquots $(0.6 \mathrm{ml})$ are taken out at precise times after injection; these aliquots are immediately injected in $6 \mathrm{ml}$ TCE at room temperature to stop the reaction.

\section{Characterization}

Powder X-ray diffraction (pXRD) patterns were recorded on a Bruker D8 DaVinci diffractometer equipped with $\mathrm{Cu}$ X-ray radiation $\left(K_{\alpha}\right.$ line, $\lambda=0.154 \mathrm{~nm}$ ) operating at $40 \mathrm{kV}$ and $40 \mathrm{~mA}$. Peak intensities were obtained by counting with the Lynxeye detector every $0.02^{\circ}$ at sweep rates of $0.5^{\circ} 2 \theta / \mathrm{min}$. Samples were placed in a PVMA sample holder with zero-background silica plates by drop-casting concentrated nanocrystal solutions. The holder was rotated at $5^{\circ}$ per minute; no background correction was applied to raw data.

Transmission electron microscopy (TEM) images were recorded on a JEOL2200FS microscope operating at $200 \mathrm{keV}$. Formvar-coated copper grids (Ted Pella, 01800-F) were used as nanocrystal supports for TEM. Thoroughly cleaned chloroform suspensions were drop-casted on the TEM grid prior to the measurements. Images were analyzed using ImageJ. ${ }^{1}$

X-ray photoelectron spectroscopy was done on a Perkin Elmer Phi 5600 ESCA system, with a $\mathrm{Mg} K_{\alpha}$ X-ray source at a take off angle of $45^{\circ}$, under ultrahigh vacuum conditions $\left(<10^{7}\right.$ torr $)$. Spectra were acquired with a pass energy of $187 \mathrm{eV}$ for survey scans or $29.35 \mathrm{eV}$ for zoomed-in scans, and a step size of $0.8 \mathrm{eV}$ for survey scans or $0.1 \mathrm{eV}$ for zoomed-in scans; the energy scale was calibrated with adventitious carbon at $284.6 \mathrm{eV}$. Samples for XPS were first precipitated out of solution and dried under nitrogen. Films were then prepared by spreading the nanocrystals powder on a $1 \mathrm{~cm}^{2}$ copper tape (Ted Pella) attached to a stainless steel holder.

Raman spectroscopy was done on a Renishaw inVia Reflex spectrometer, equipped with a confocal microscope connected to a CW diode-pumped solid-state laser with a fundamental emission at $532 \mathrm{~nm}$. The sample was positioned under the laser light using a motorized stage. The stage position was controlled and the spectral data were acquired with the commercial WiREInterface software. This software allows for control over the laser power, exposure time, spectral range covered, and stage positioning for mapping of a sample. The parameters used were a laser power of $1 \mathrm{~mW}$ at the sample and an integration time of $3 \mathrm{~s}$. Each spectrum was generated from an average of five hundred spectral acquisitions.

UV-vis-NIR absorption spectra were measured on a Olis 17 spectrometer from 2,500 $\mathrm{nm}$ to $500 \mathrm{~nm}$ at a rate of $103 \mathrm{~nm} / \mathrm{min}$, and three reads per datum, 2,000 increments. $1 \mathrm{~cm}$ infrasil cuvettes in both reference (filled with pure solvent only) and sample beam paths were used. All data were acquired with constant signal-to-noise ratio across the whole spectral range by letting the instrument vary the slits width during the acquisition to maintain a constant detector voltage; the bandwidth varied from $0.5 \mathrm{~nm}$ to $1.0 \mathrm{~nm}$ across the spectral range. 
3. TEM images and size-distribution analyses

a) As-prepared sample

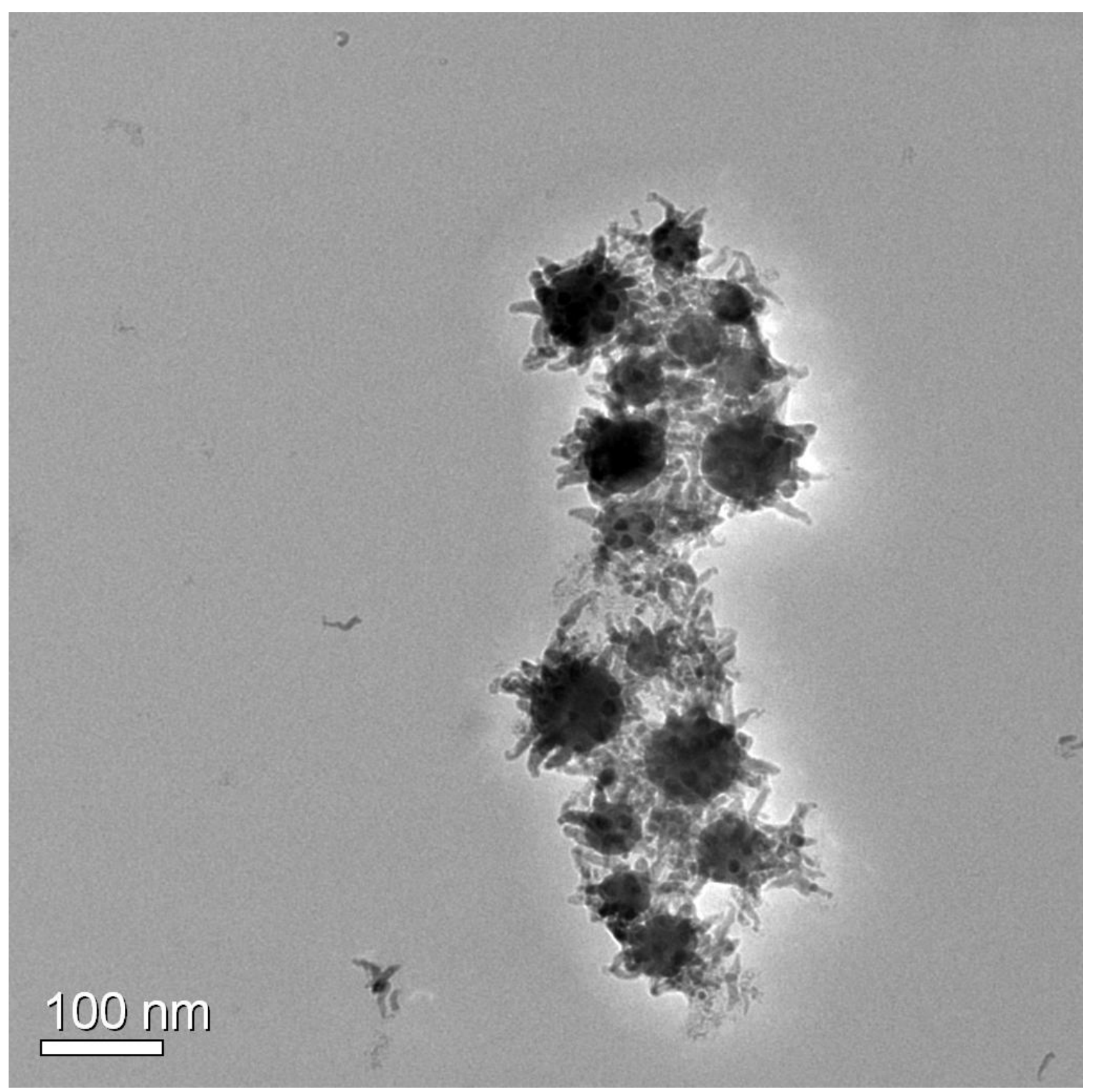

Figure S 1 


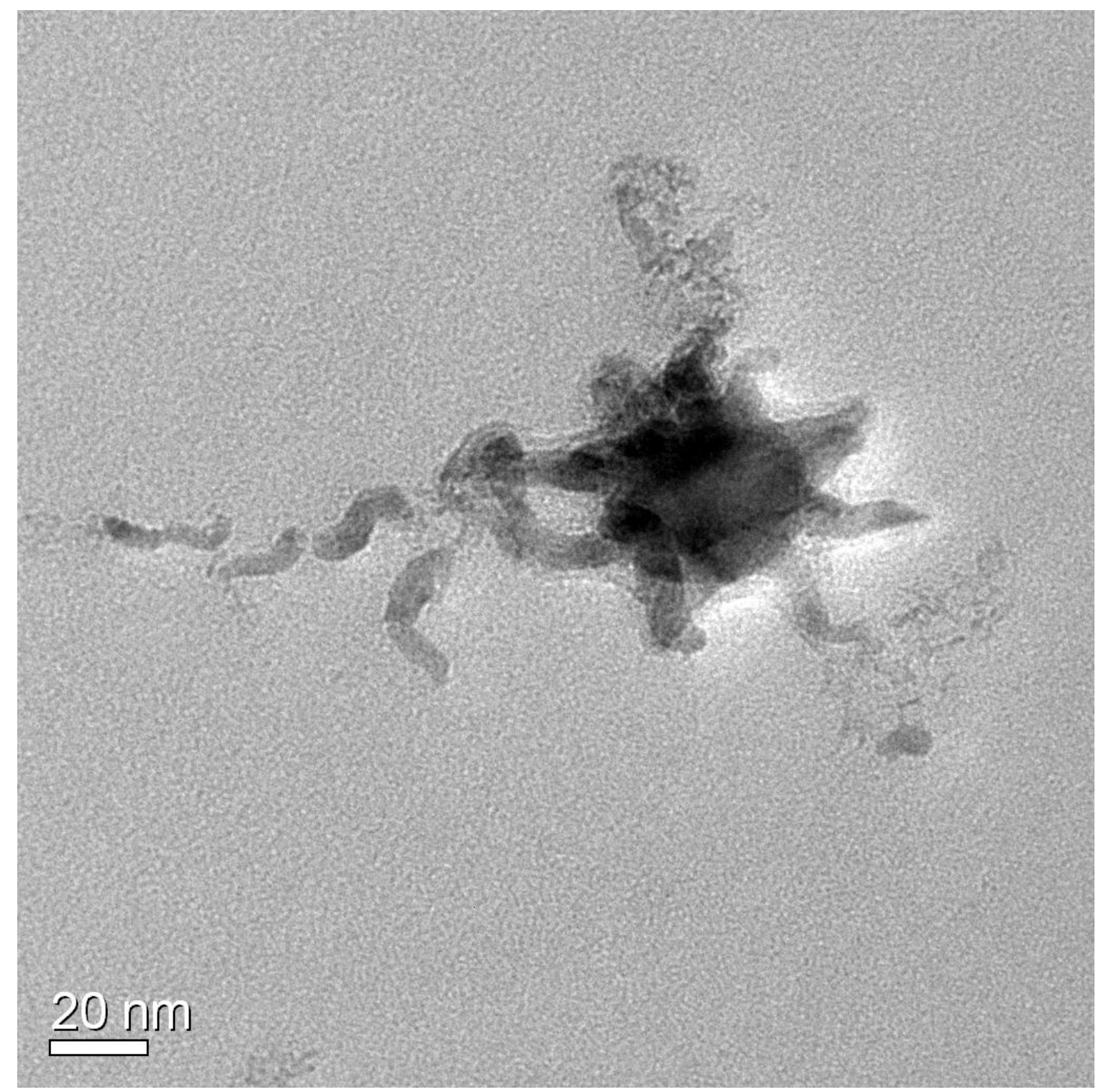

Figure S 2 


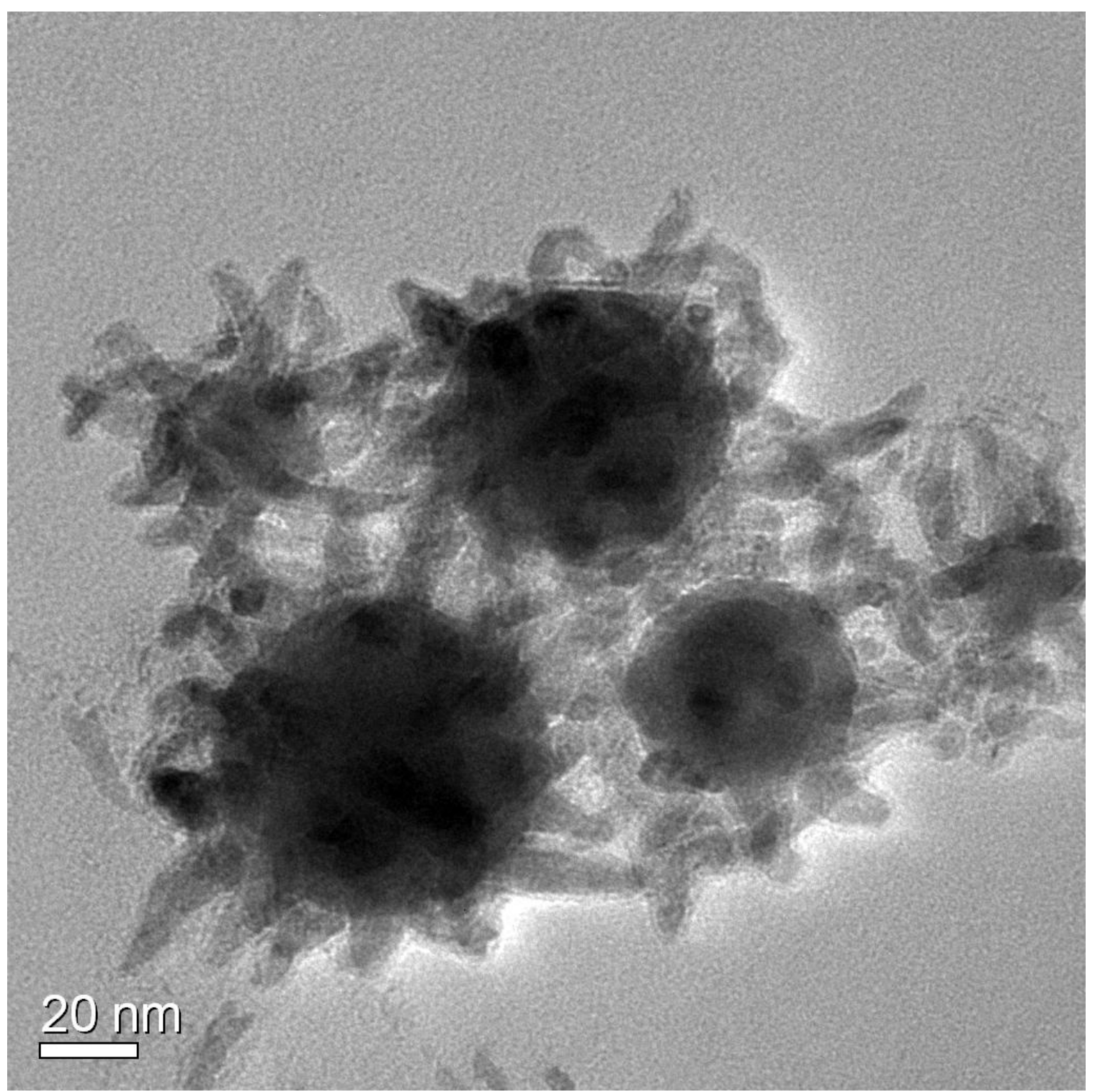

Figure S 3 


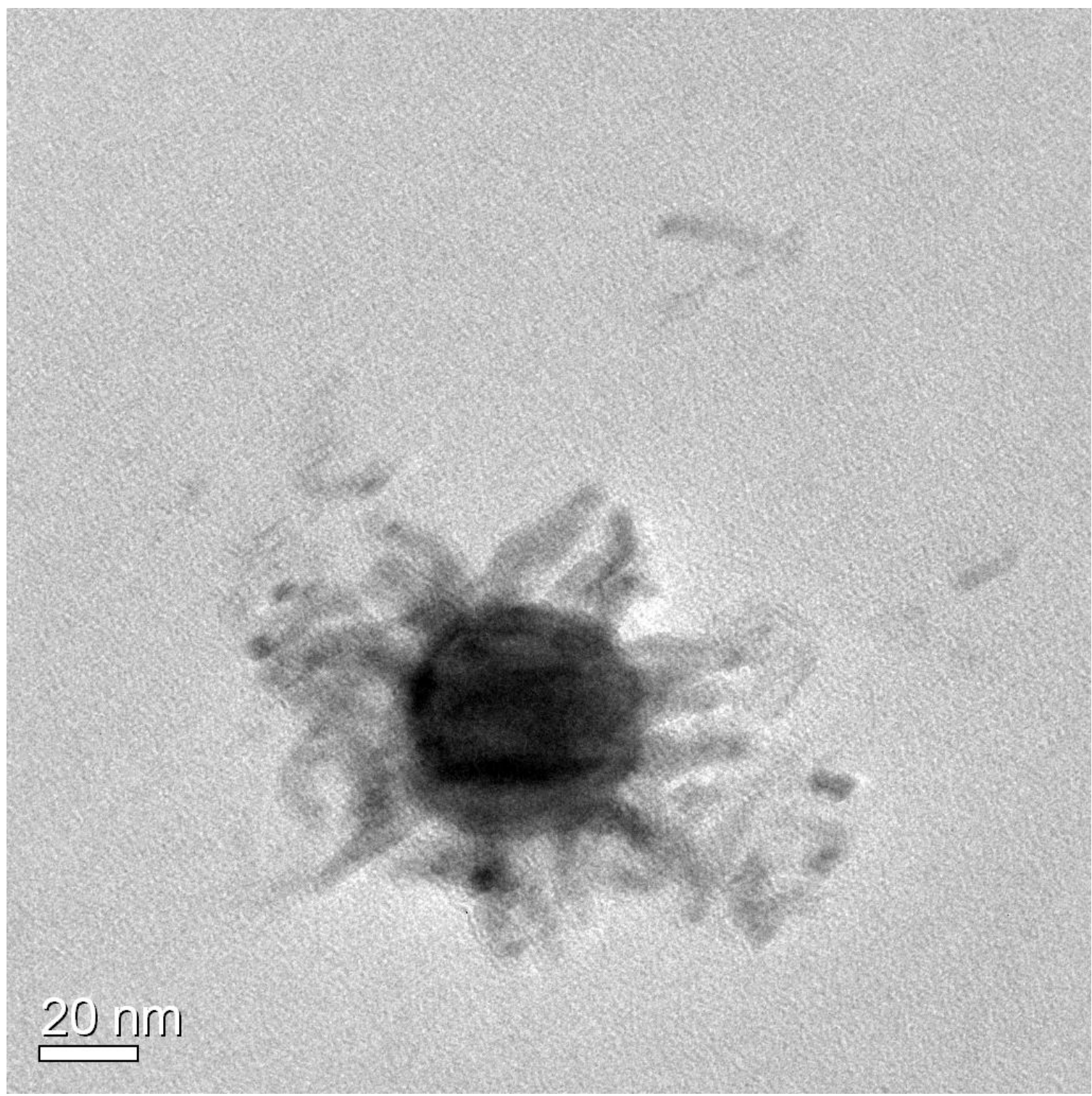

Figure S 4 


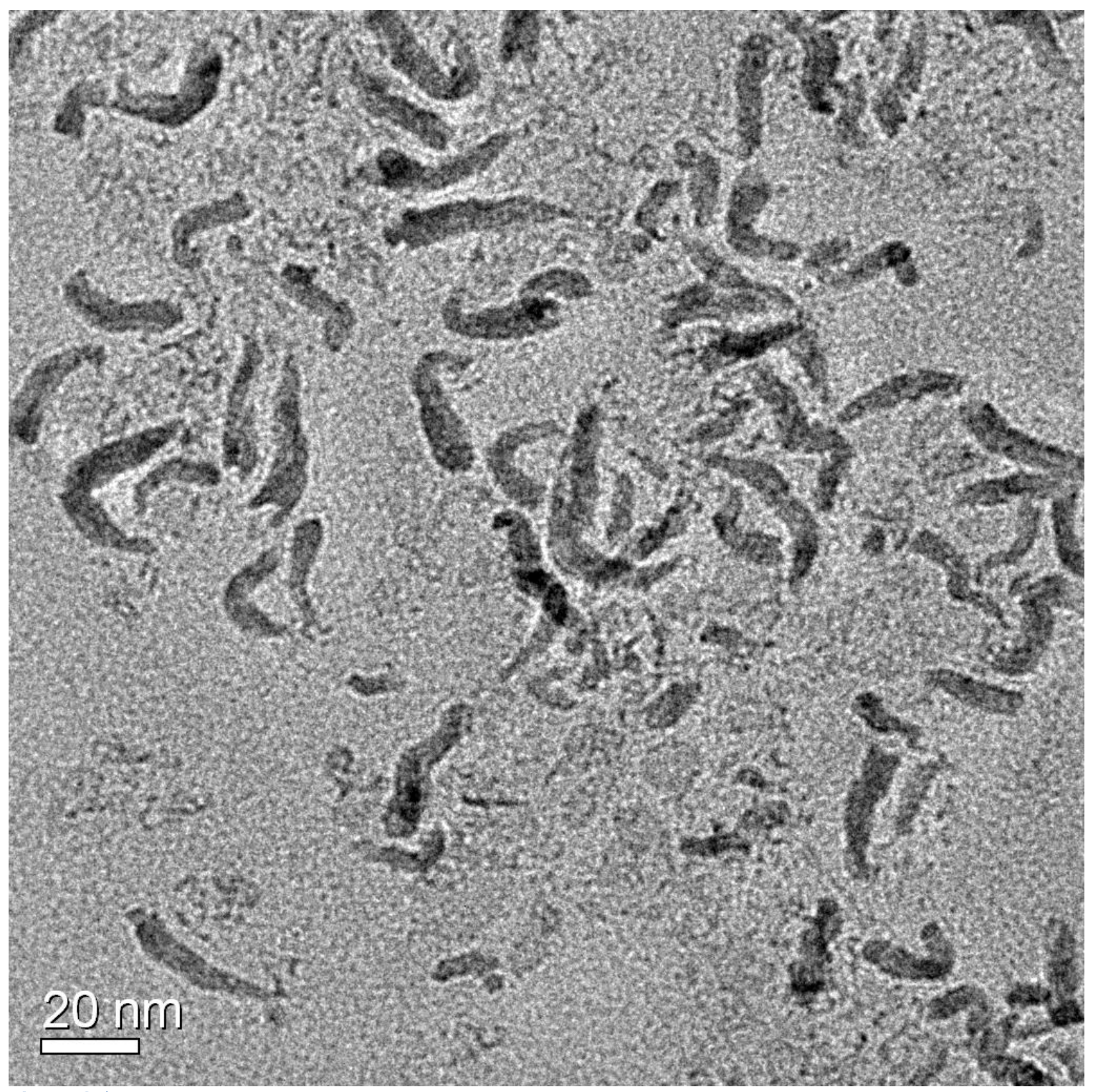

Figure S 5 


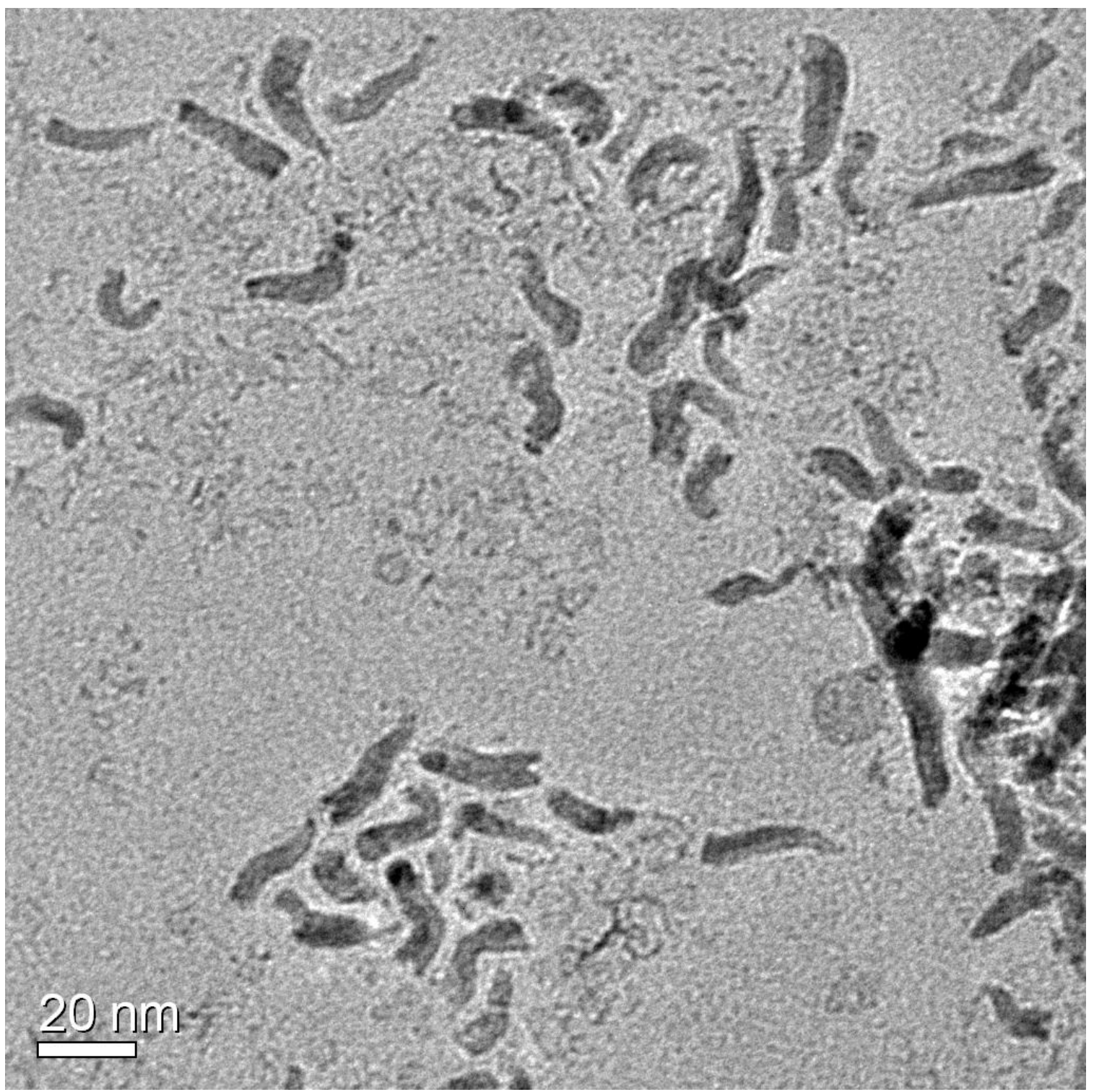

Figure S 6 


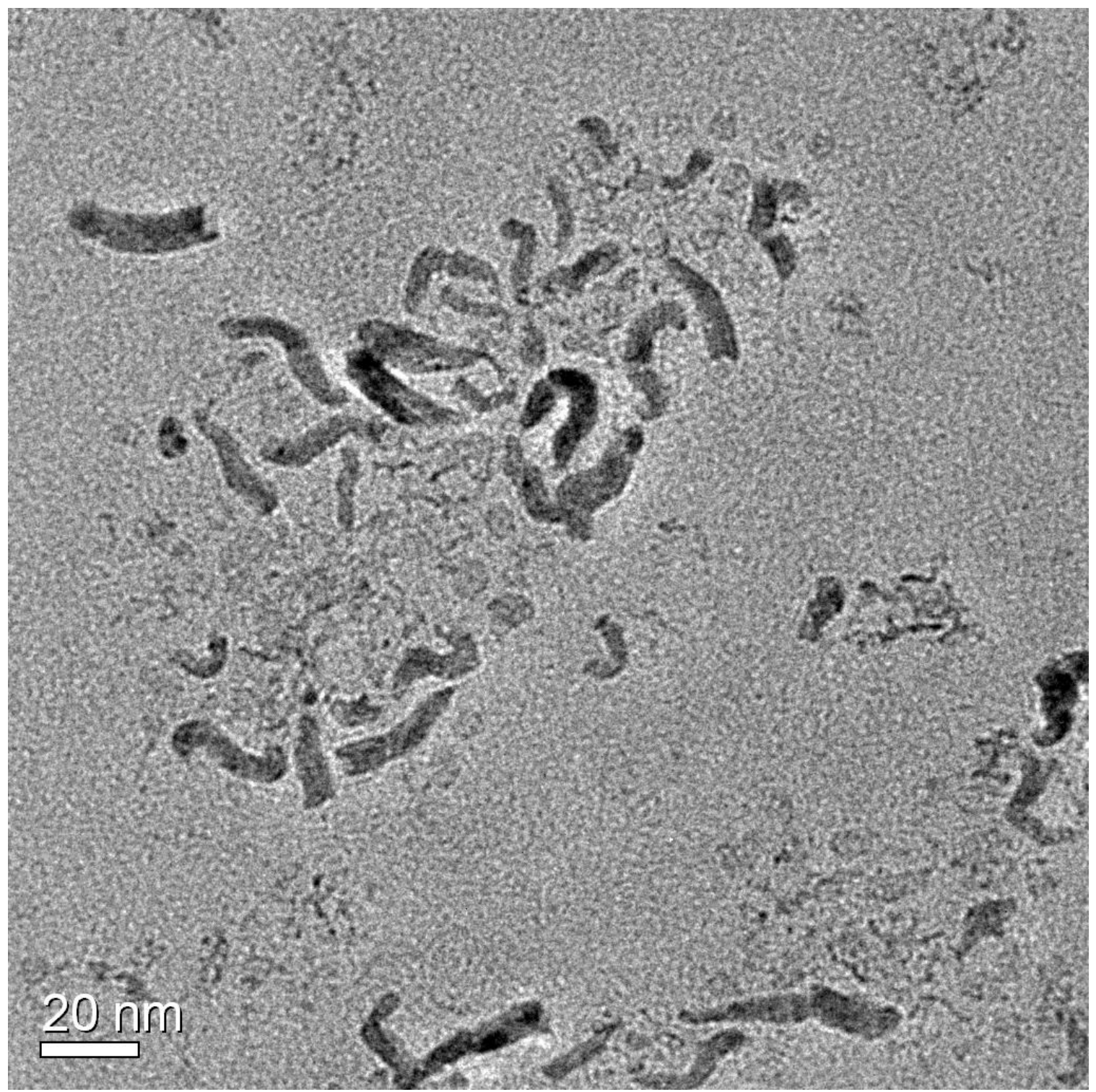

Figure S 7 


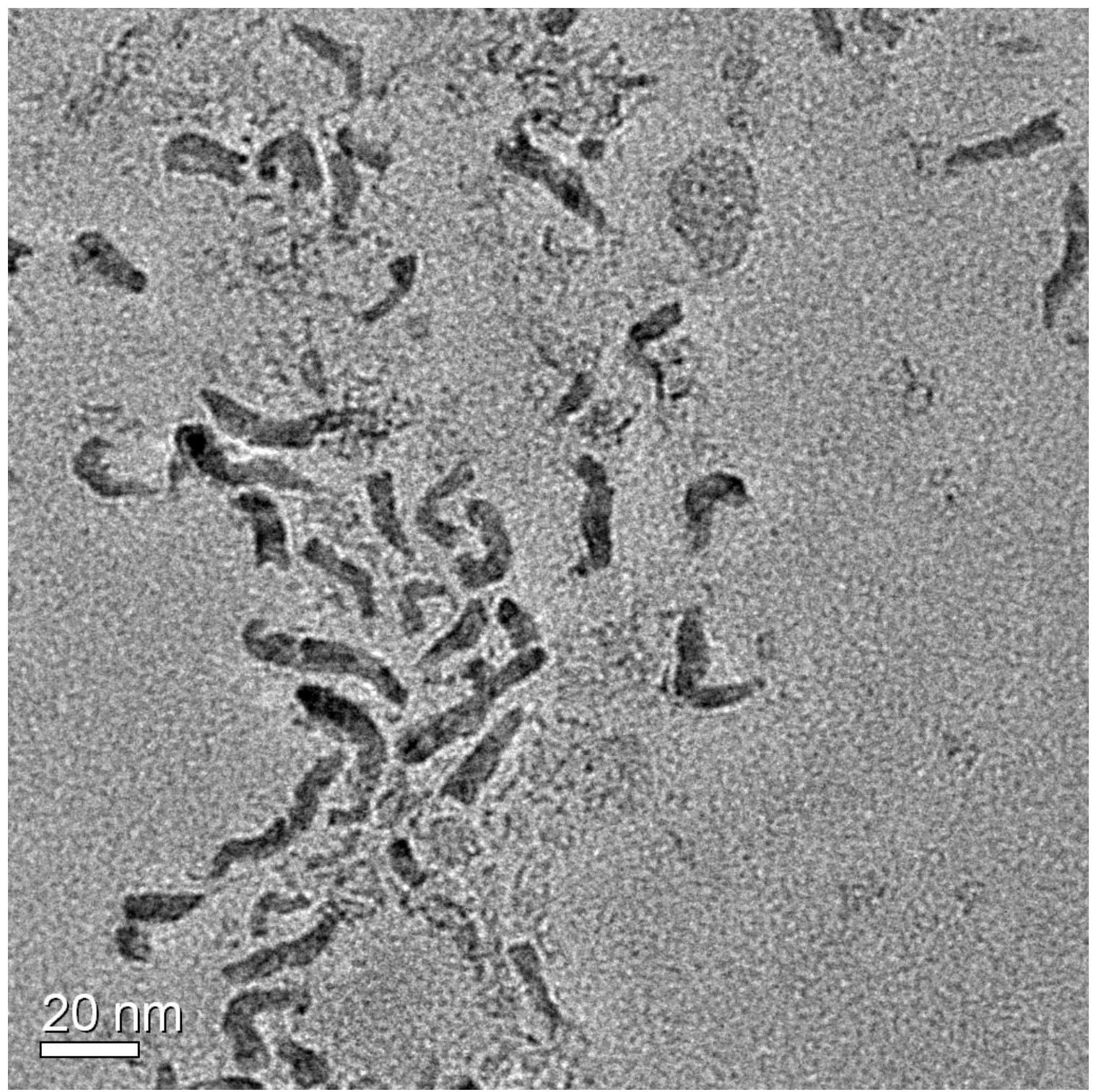

Figure S 8 

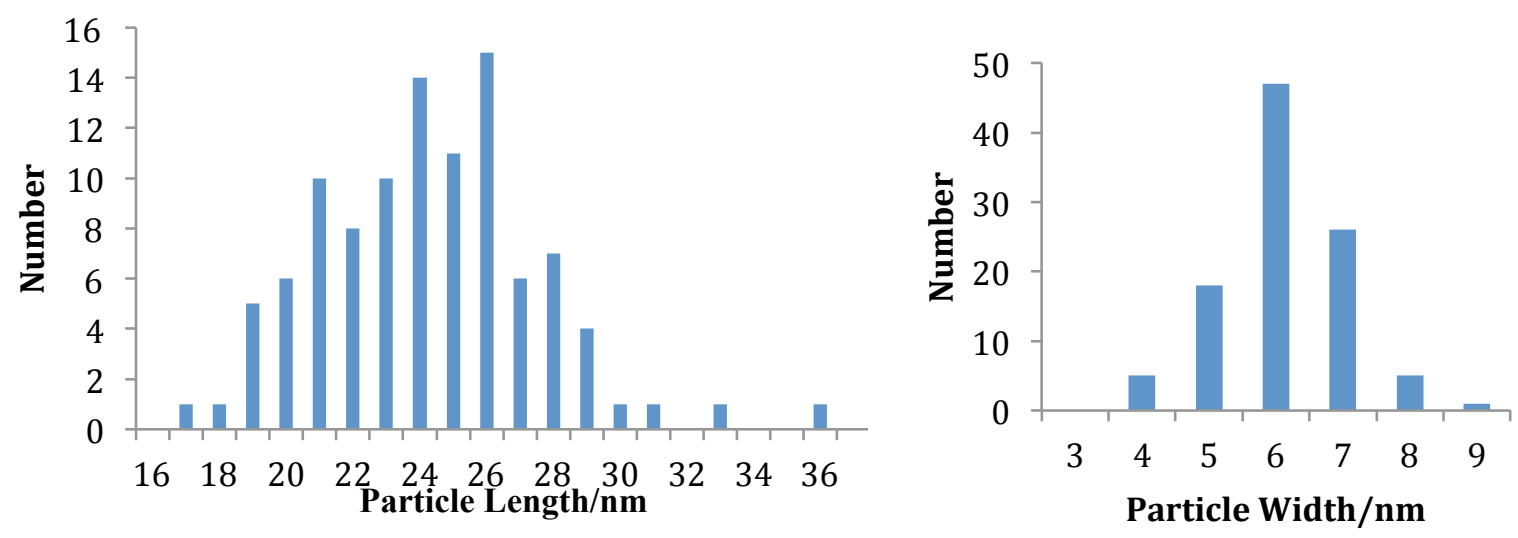

Figure S 9 - Size Distribution of the as-prepared sample.

Table S1 - Statistical Analysis of the Distribution shown in Fig. S9 ( $N=102$ particles)

\begin{tabular}{|l|l|l|l|}
\hline & Length $(\mathrm{nm})$ & Width $(\mathrm{nm})$ & Aspect Ratio \\
\hline Average & 23.8 & 5.6 & 4.3 \\
\hline Standard deviation & 3.3 & 0.9 & 0.8 \\
\hline Median & 23.8 & 5.6 & 4.3 \\
\hline
\end{tabular}


b) Acid-Treated Sample

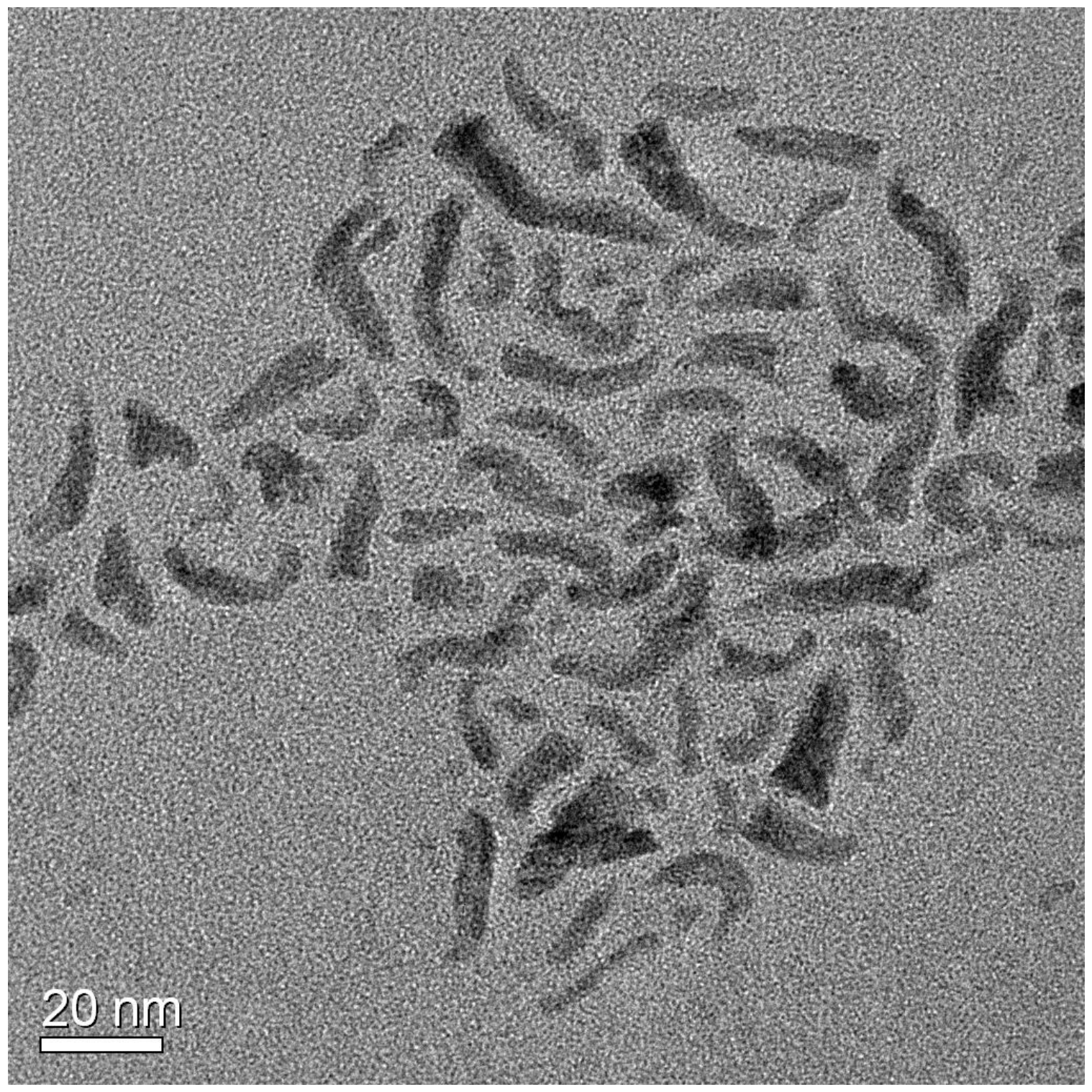

Figure S 10 


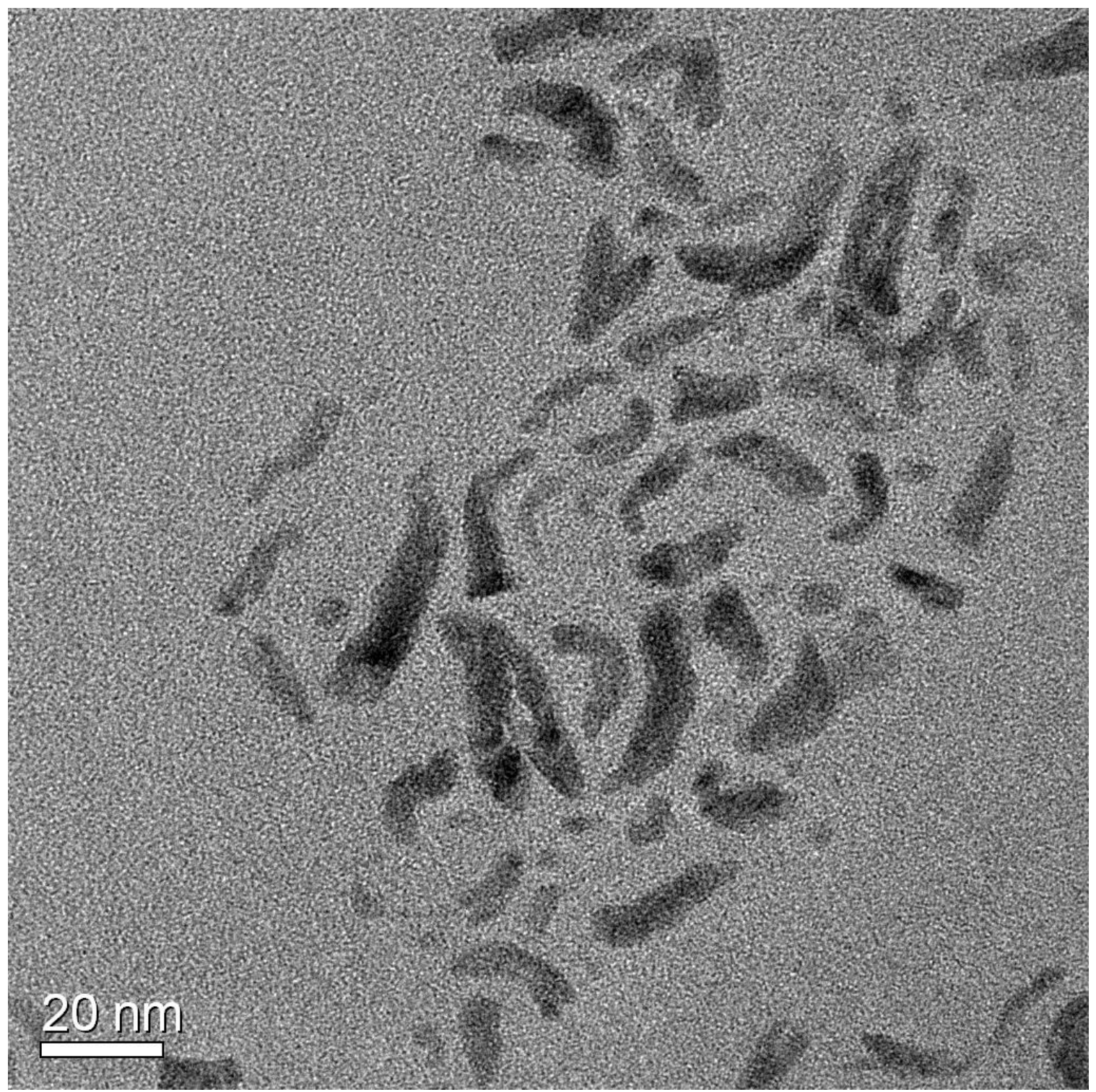

Figure S 11 


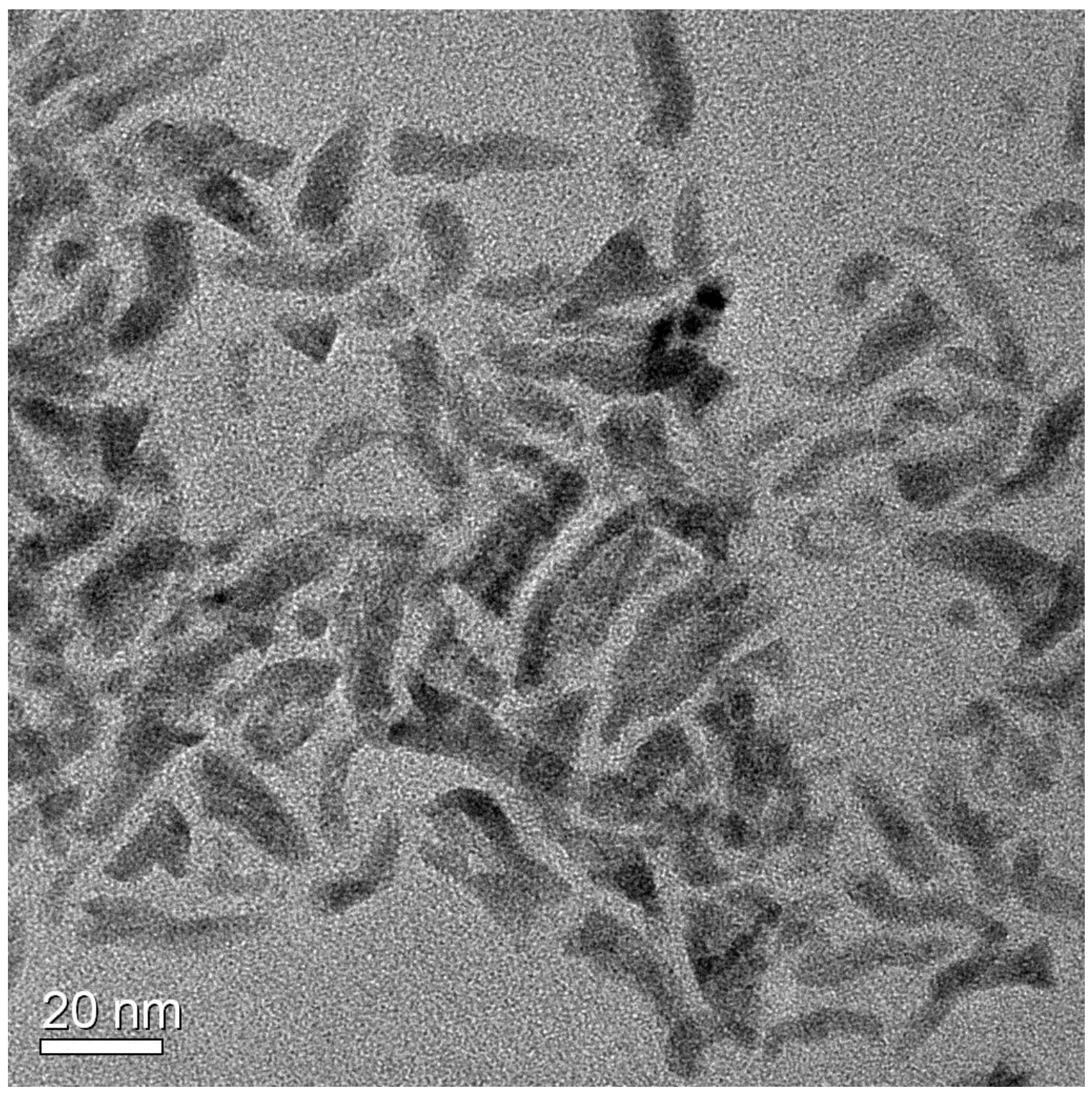

Figure S 12 


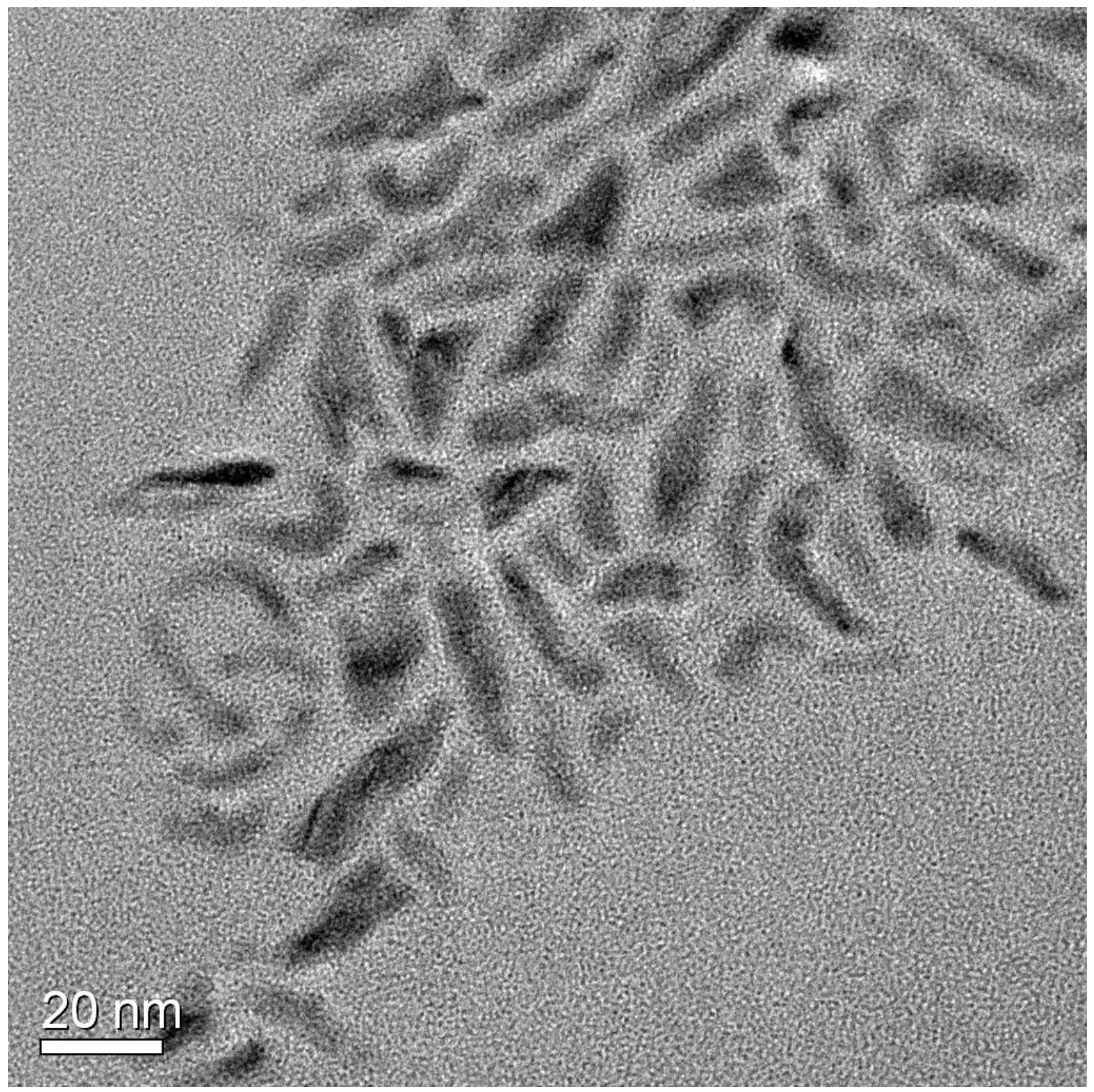

Figure S 13 


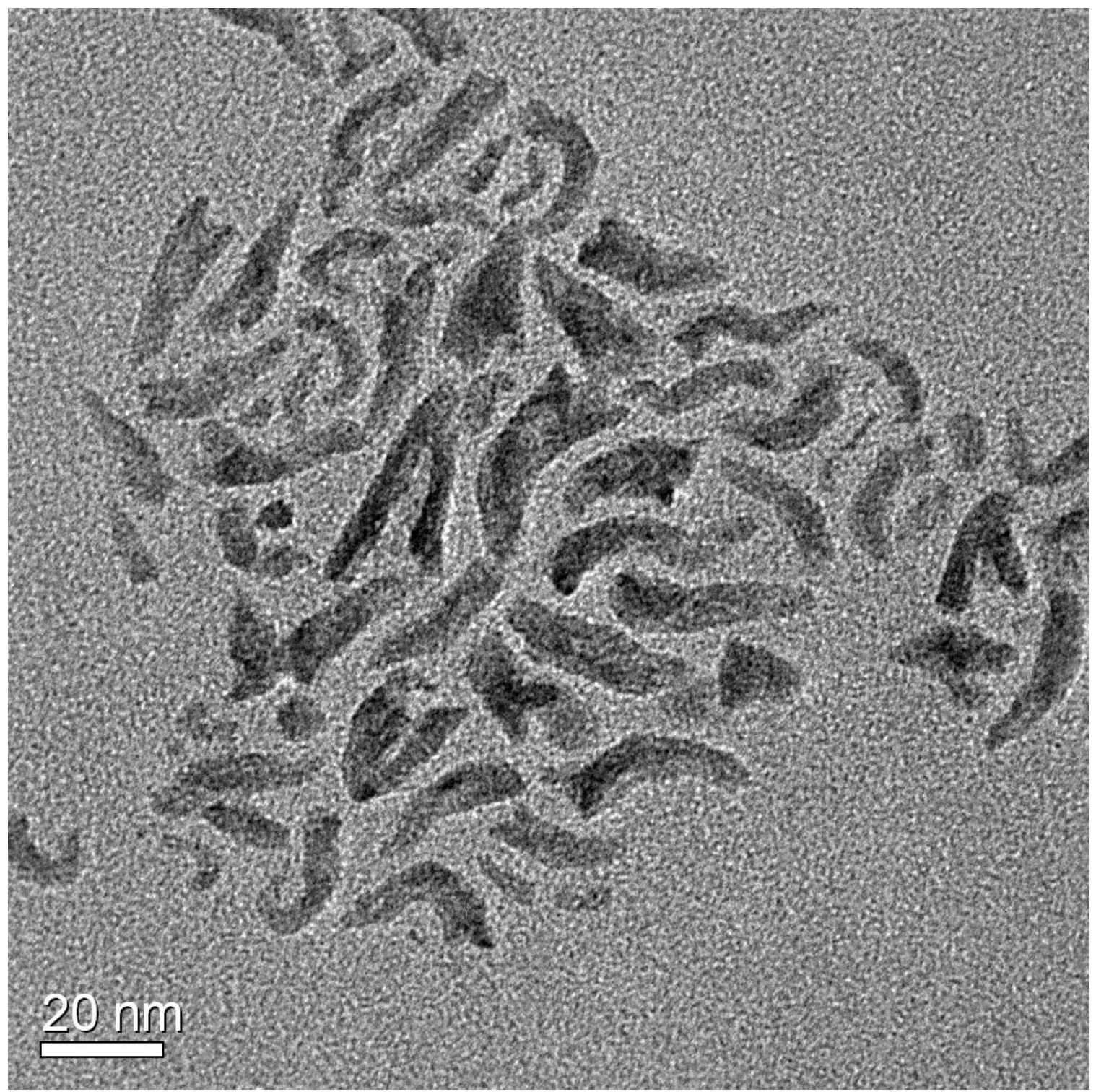

Figure S 14 


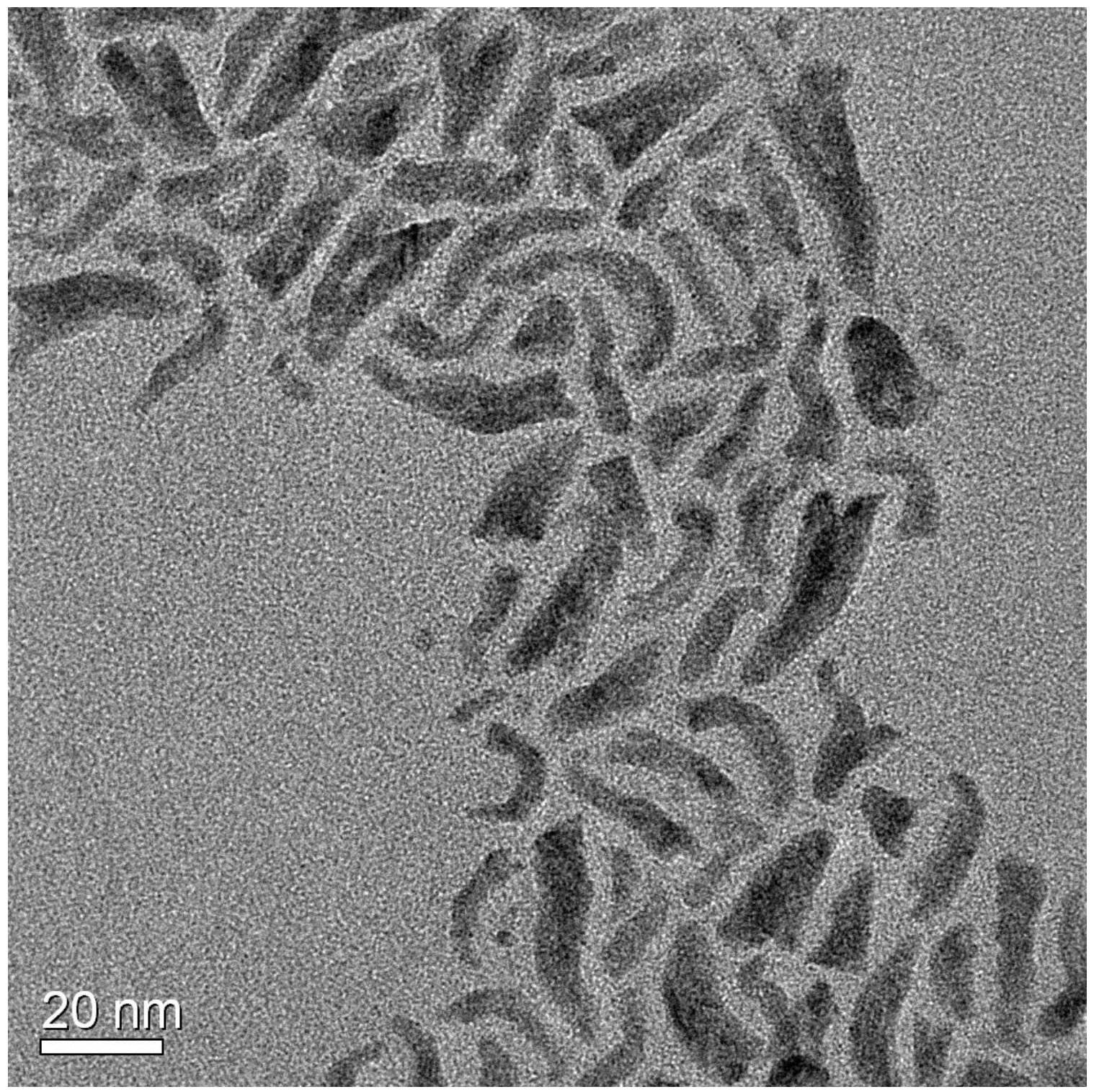

Figure S 15 


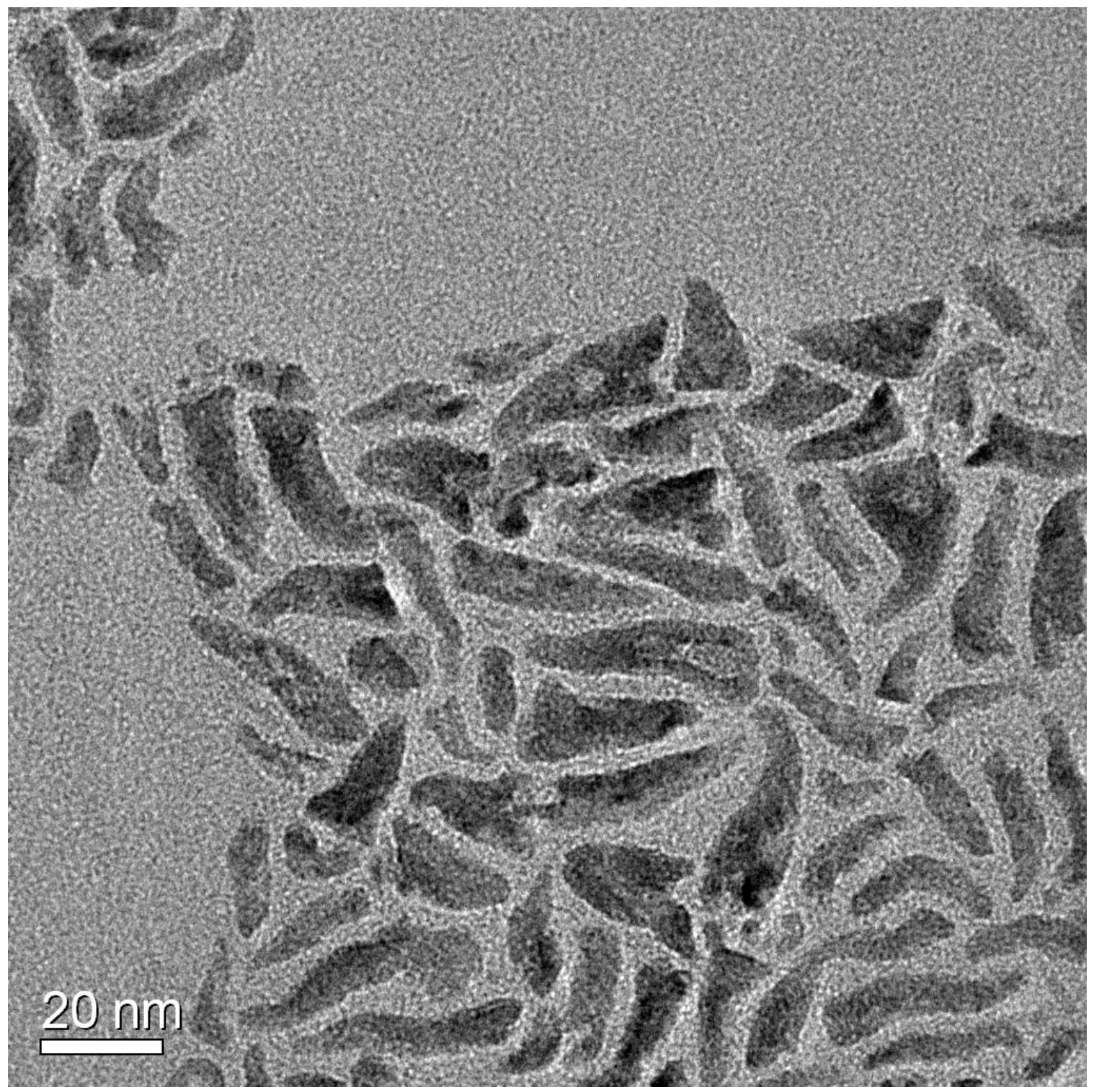

Figure S 16 


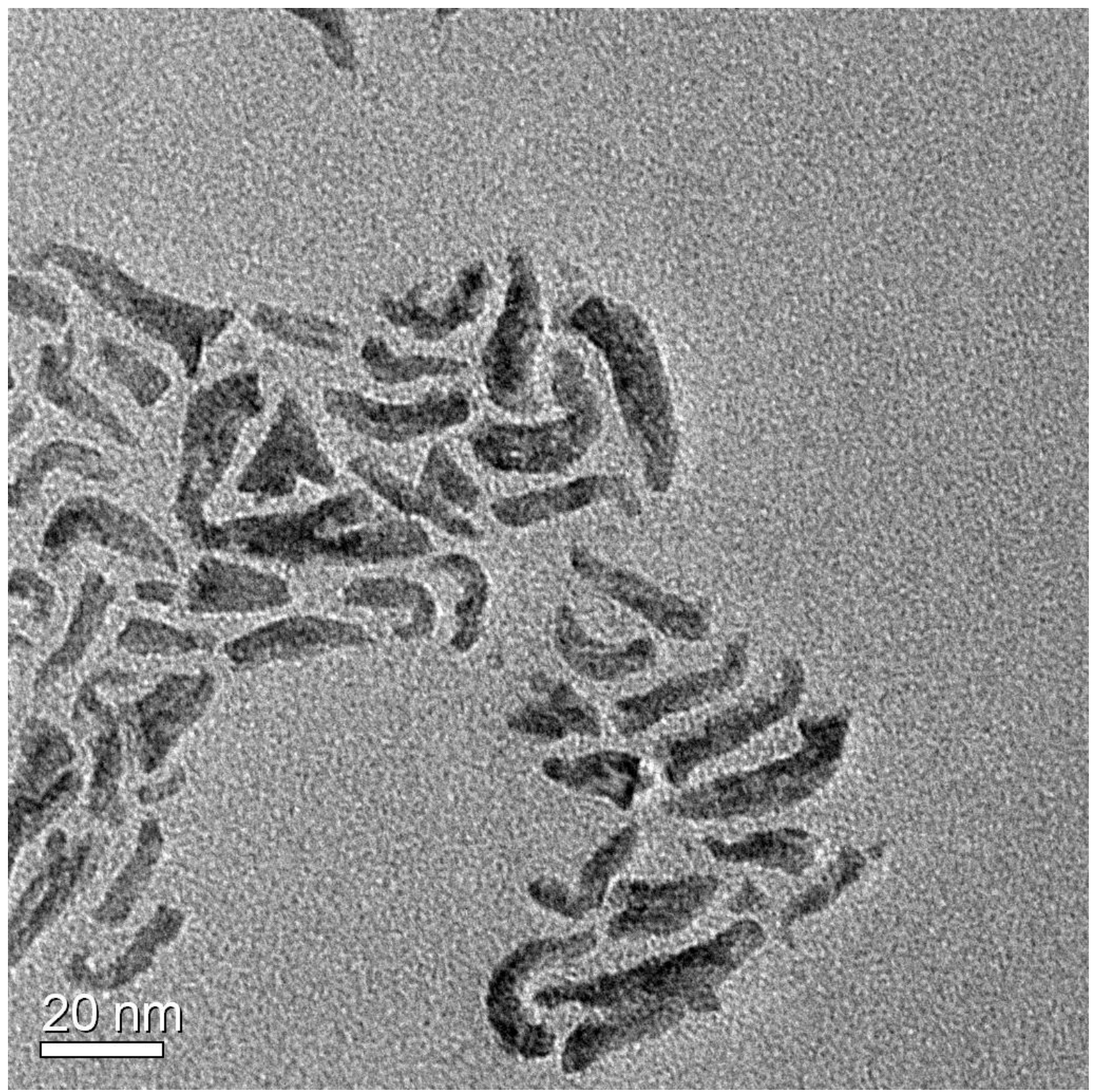

Figure $\mathbf{S} 17$ 


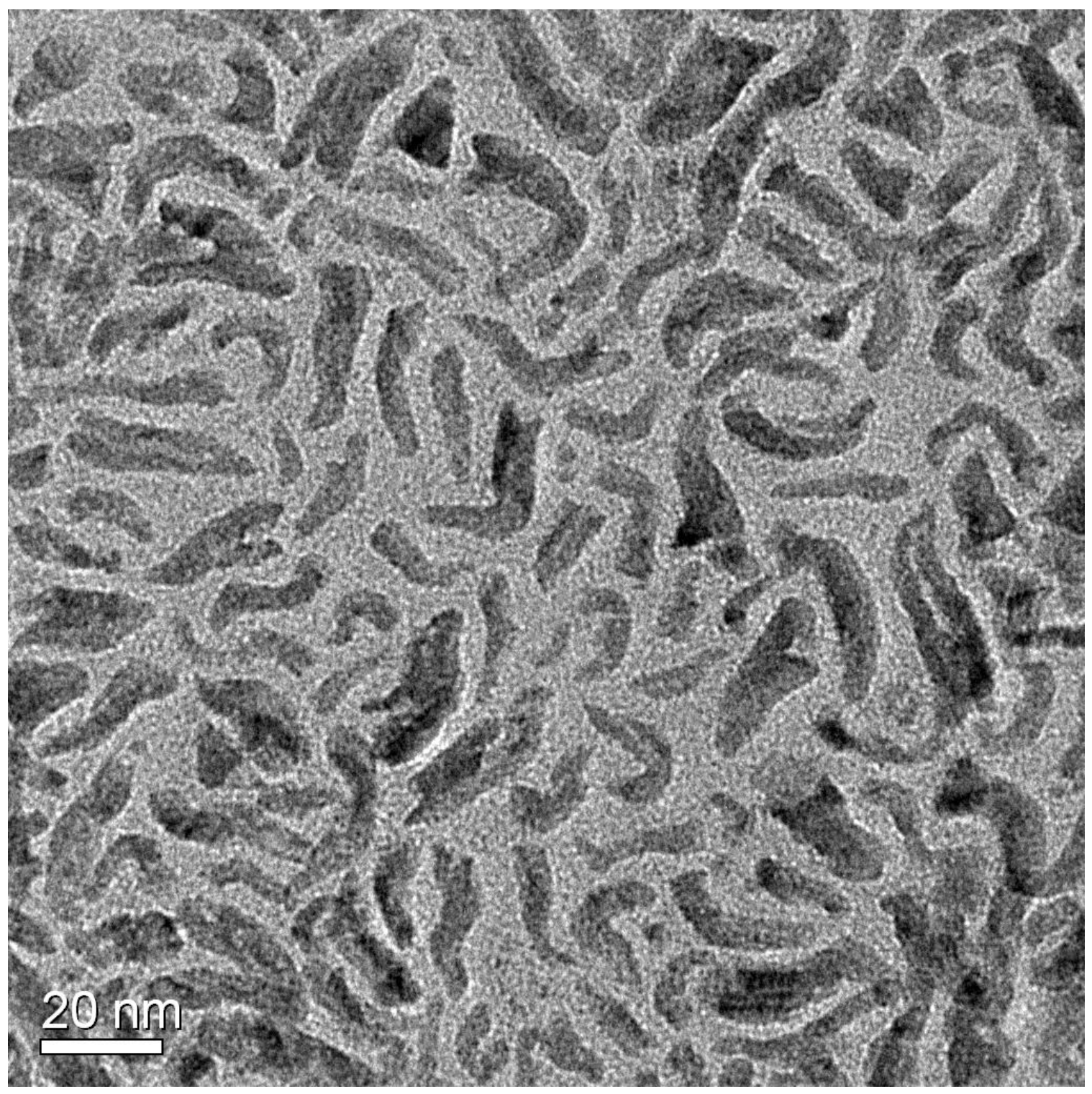

Figure S 18 


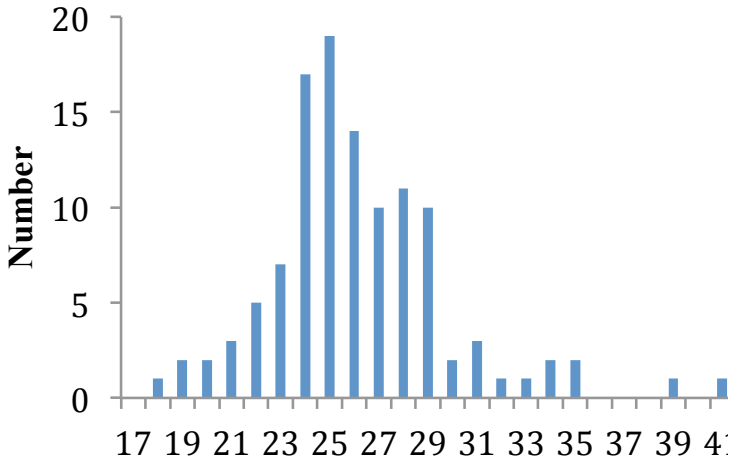

Particle Length/nm

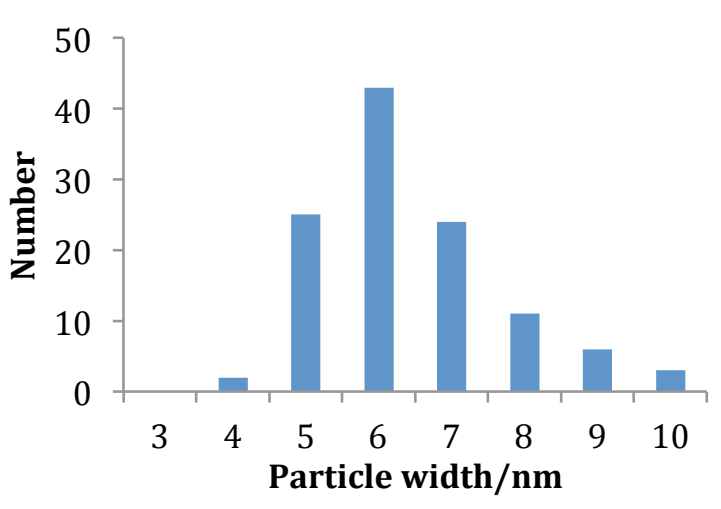

Figure S 19 = Size Distribution of the acid-treated sample

Table S2 - Statistical Analysis of the Distribution shown in Figs. S18 ( $N=114$ particles)

\begin{tabular}{|l|l|l|l|}
\hline & Length $(\mathrm{nm})$ & Width $(\mathrm{nm})$ & Aspect Ratio \\
\hline Average & 25.7 & 5.9 & 4.5 \\
\hline Standard deviation & 3.6 & 1.2 & 0.9 \\
\hline Median & 25.4 & 5.7 & 4.5 \\
\hline
\end{tabular}


c) InN nanorods synthesized from lithium bis(trimethylsilyl)amide (LiHMDS)

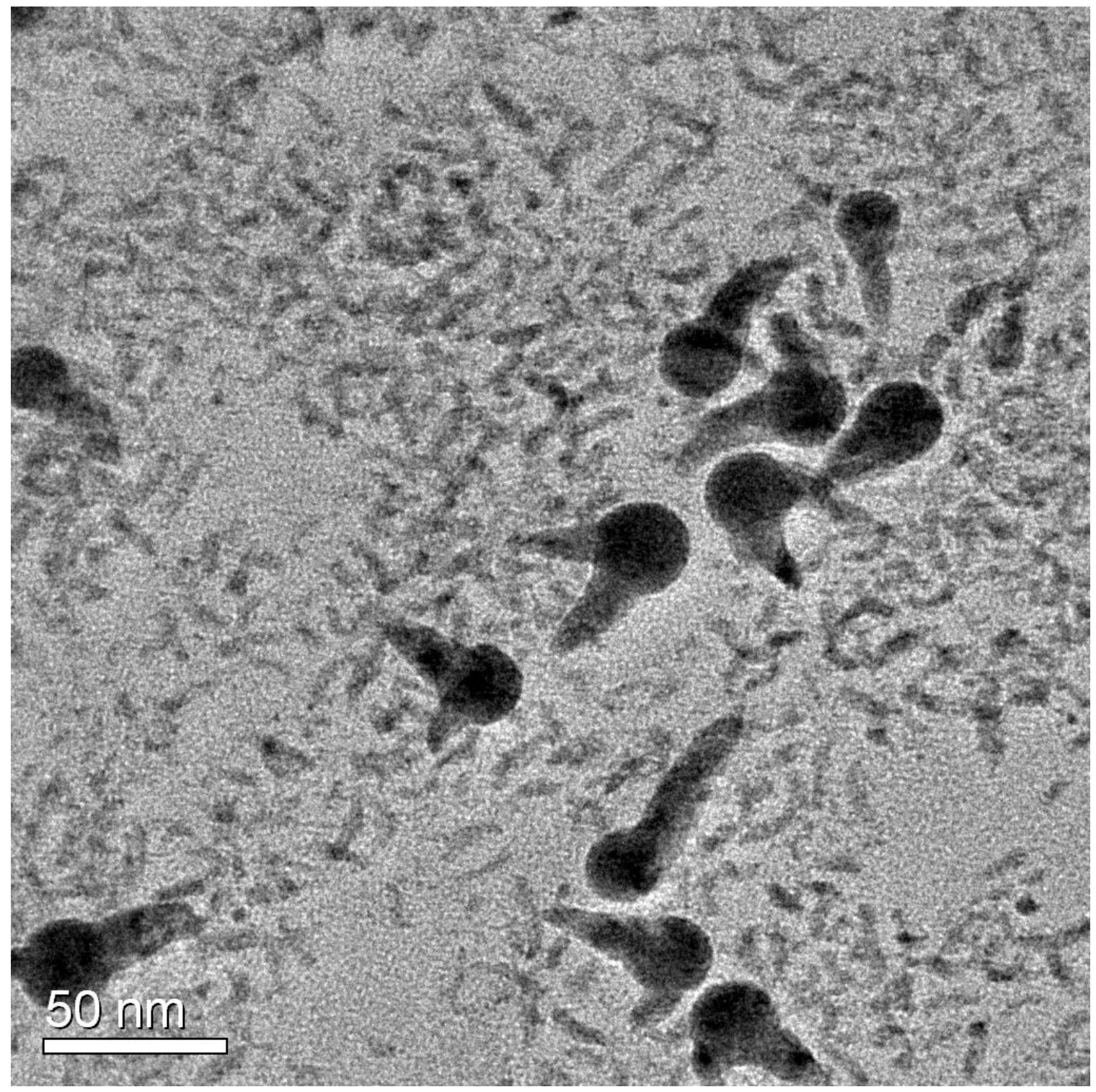

Figure S 20 


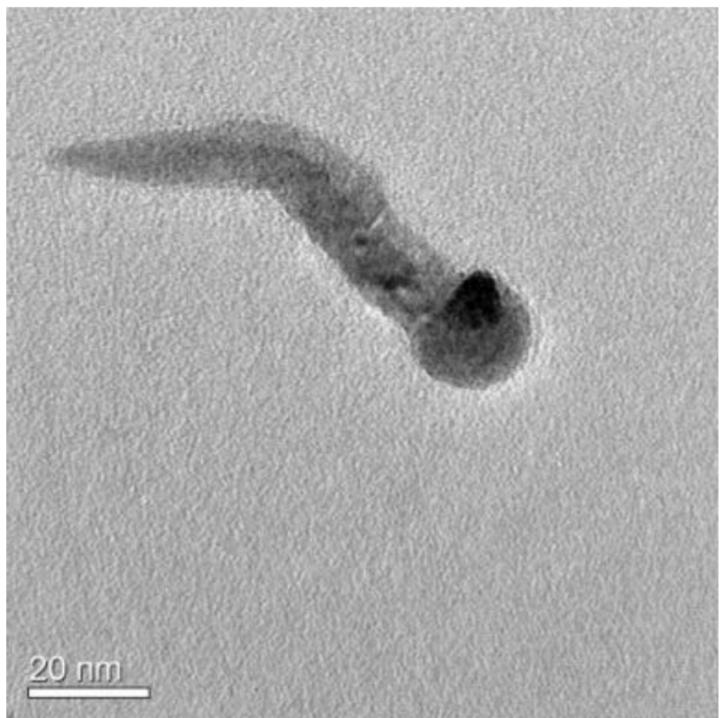

(a)

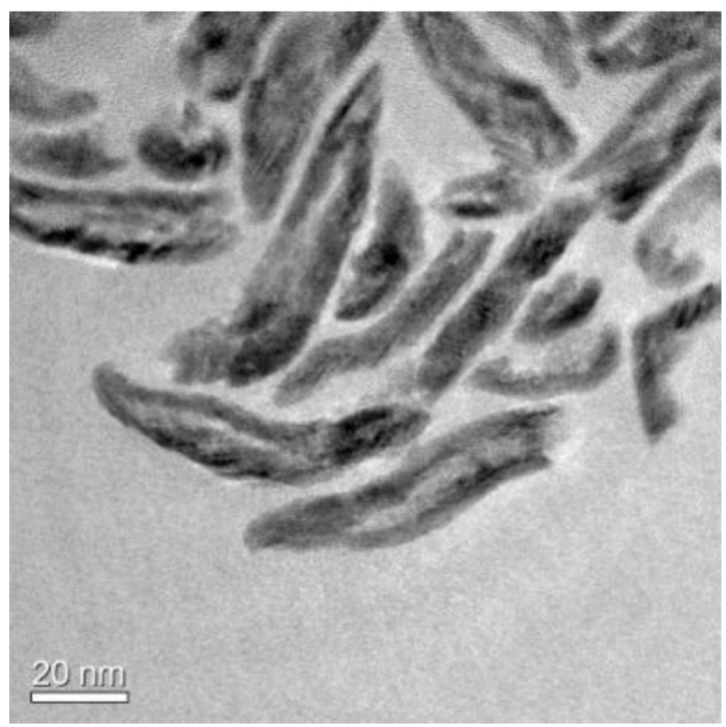

(c)

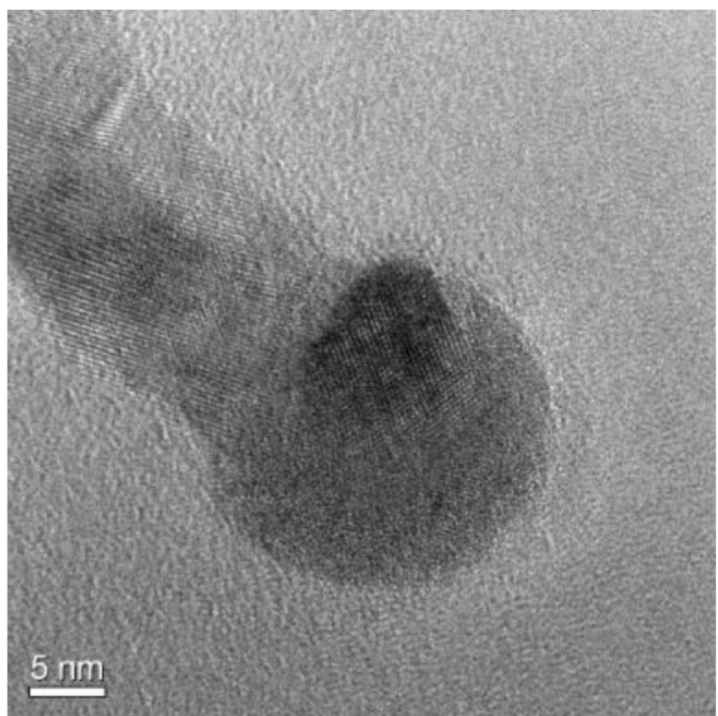

(b)

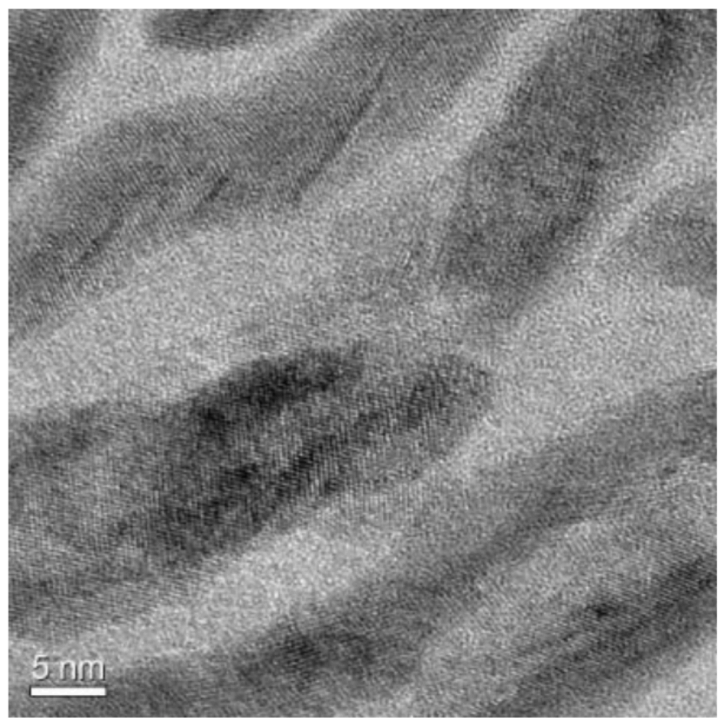

(d)

Figure S21- (a,b): As-prepared; (c,d): acid-treated. 
4. XPS Data
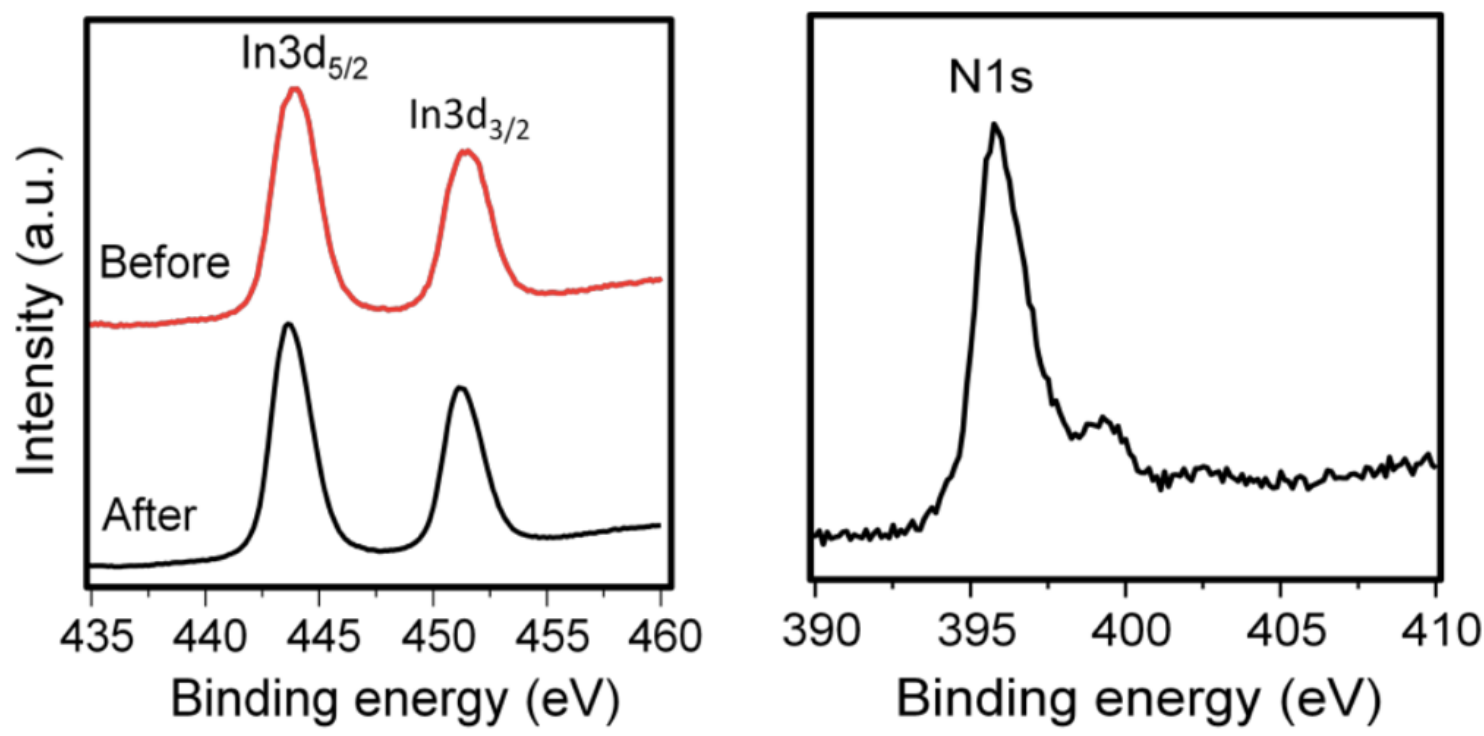

Figure S 22 - XPS data of the as-prepared (before) and acid-treated (after) samples

5. Raman Data

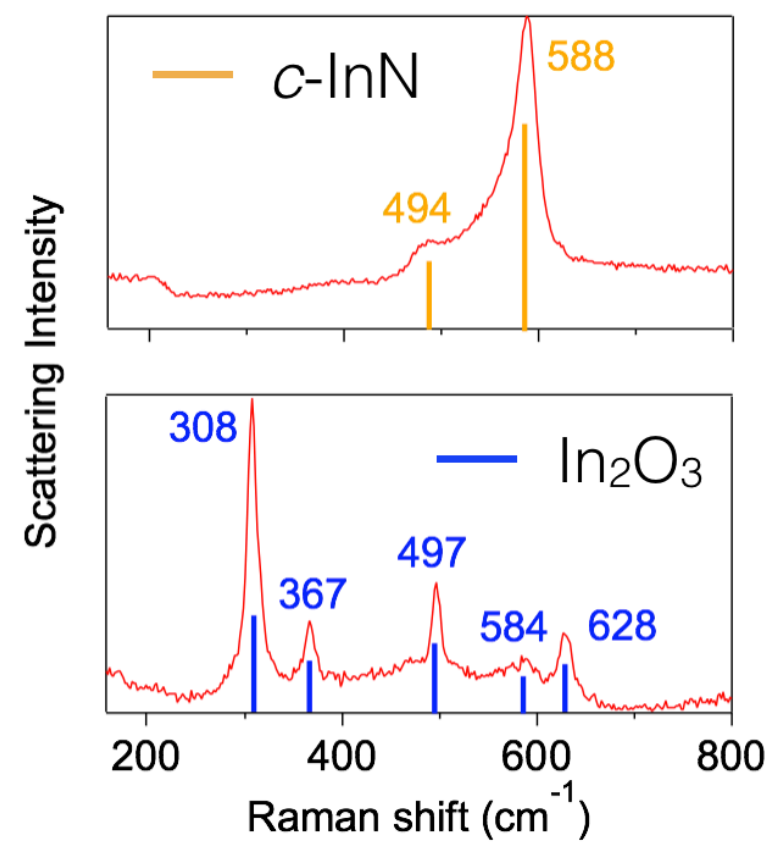

Figure S 23 - Raman spectra of the acid-treated sample before (top) and after (bottom) heating at $500{ }^{\circ} \mathrm{C}$ overnight, which converts the nitride into an oxide; the numbers listed correspond to the phonon energies of the bulk materials, respectively. ${ }^{2,3}$ 
6. Absorption data - comparison with $\mathrm{NaNH}_{2}$ synthesis ${ }^{4}$

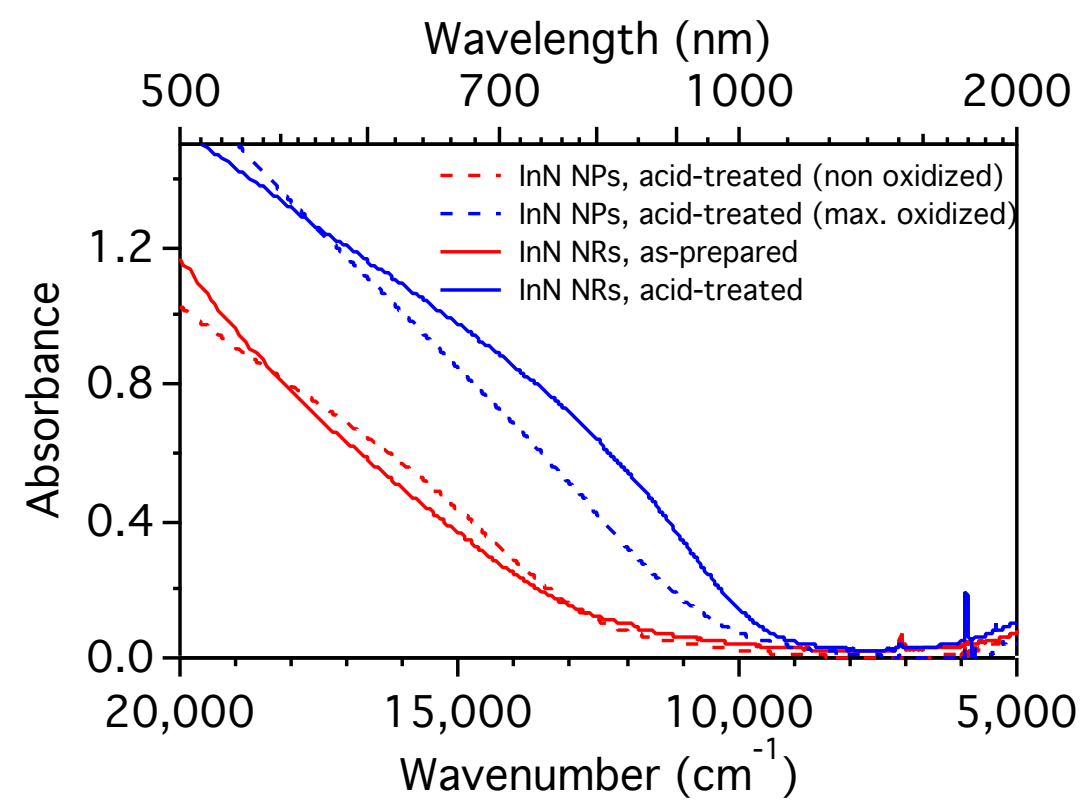

Figure S 24 - Absorption spectra comparing InN nanorods (NRs) as synthesized here, before and after acid treatment, with $6 \mathrm{~nm}$ InN (BF-"-capped) nanoparticles (NPs) synthesized using the conventional $\mathrm{NaNH}_{2}$ approach. ${ }^{4}$ The oxidation is performed exactly as described by Palomaki et al., ${ }^{4}$ using $\mathrm{NOBF}_{4}$ as the oxidant on $\mathrm{BF}_{4}-$-capped NPs.

7. EDS data of the $\operatorname{InN} / \operatorname{In}(0)$ sample

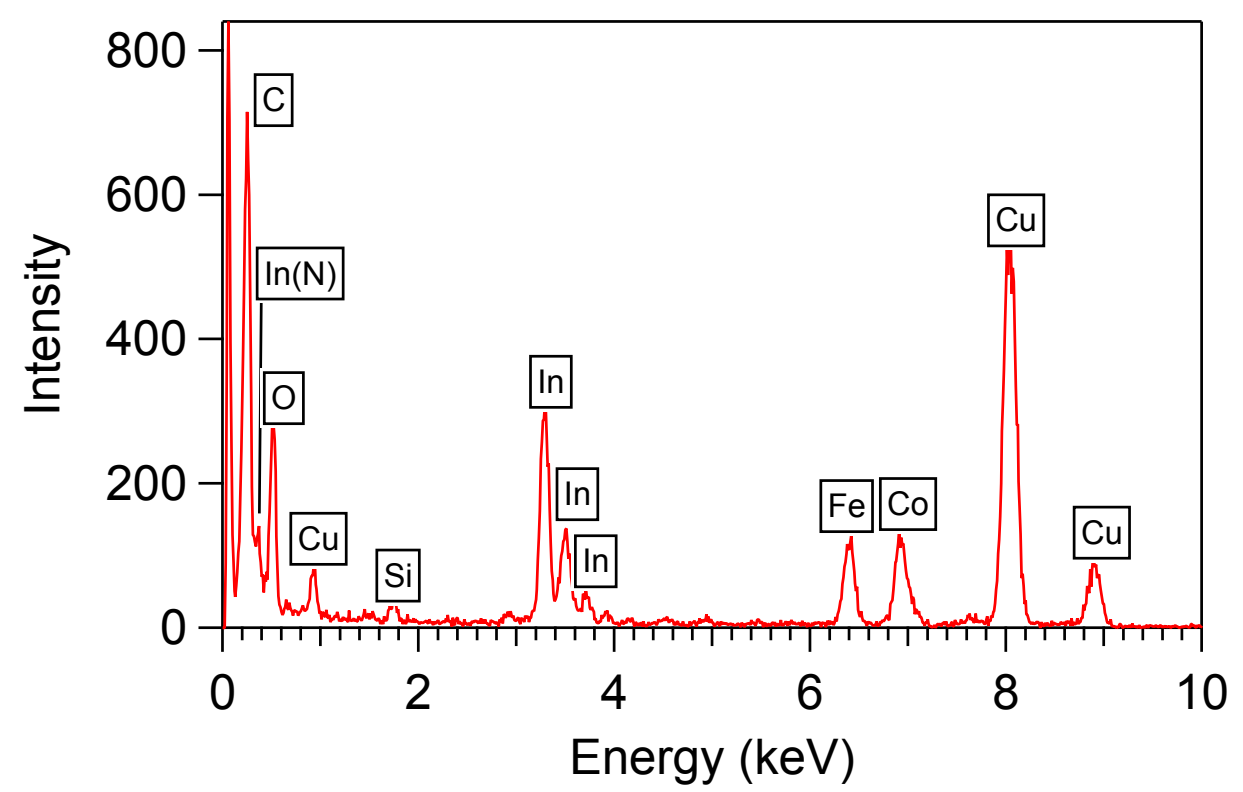

Figure S 25 - Energy-Dispersive X-Ray Spectroscopy of the as-synthesized InN/In(0) sample. Unfortunately, the EDS data for InN cannot be used to confirm the presence (or absence) of nitrogen, as the nitrogen peak in EDS (around $390 \mathrm{eV}$ ) overlaps directly with the indium M-series X-rays (around 450 $\mathrm{eV}$ ); given the resolution of about $150 \mathrm{eV}$ typical for EDS, one cannot distinguish the two species directly with that method. Another issue with EDS is the notoriously weak intensity of nitrogen X-rays, which are hard to detect even in the absence of overlapping bands due to efficient Auger processes that reduce the efficiency of the X-ray emission. 


\section{References}

(1) Rasband, W. S., ImageJ, National Institutes of Health, Bethesda, Maryland, USA, http://imagej.nih.gov/ij/, 1997-2016.

(2) Kaczmarczyk, G.; Kaschner, A.; Reich, S.; Hoffmann, A.; Thomsen, C.; As, D. J.; Lima, A. P.; Schikora, D.; Lischka, K.; Averbeck, R.; Riechert, H. Appl. Phys. Lett. 2000, 76, 2122-2124.

(3) Olivia, M. B.; Ariano, D. R.; Cleocir, J. D.; Alexandre, J. C. L.; Edson, R. L.; Adenilson, J. C. J. Phys. D: Appl. Phys. 2010, 43, 045401.

(4) Palomaki, P. K. B.; Miller, E. M.; Neale, N. R. J. Am. Chem. Soc. 2013, 135, 14142-14150. 